\title{
The integration Effect between Mineral and bio- fertilization on Wheat Production in Soils High in Iron at the New Valley, Egypt
}

\author{
H. A. Fawy, S. M. Ibrahim, H. K. Abo EL-Ela and Noha M. Abd \\ EL- Hameed \\ Soil Fertility and Microbiology Dept., Desert Research Center (DRC), \\ Cairo, Egypt.
}

\begin{abstract}
I IGH Fe content in the New Valley soils hinders the absorption of Doth $\mathrm{P}$ and $\mathrm{Zn}$ by plants, which is negatively reflected on crop production. Field experiments were carried out in two successive seasons at two sites under the conditions of the New Valley soils in order to increase wheat yield by the integration between mineral $(\mathrm{P}$ and $\mathrm{Zn}$ ) and bio-fertilization (Mycorrhiza and Azotobacter). The first experiment was carried out at El-Monera (sandy soil), El-Kharaga Oasis, while the second one was carried out at Al-Kaser (clayey soil), El-Dakhlla Oasis. Grains of wheat variety Sakha 93 was cultivated in both locations. Application of mineral $\mathrm{P}$ and $\mathrm{Zn}$ fertilizers along or with bio-fertilizers increased wheat yield parameters, nutrients content, total antioxidants, and phenols of wheat. Moreover, increasing the application rates of $\mathrm{P}$ and $\mathrm{Zn}$ decreased $\mathrm{Fe}$ and $\mathrm{Mn}$ contents in wheat and increased $\mathrm{N}, \mathrm{K}, \mathrm{Zn}$ and $\mathrm{Cu}$ contents in the straw and grains. The most effective treatment for yield, nutrients and biochemical contents was by applying $\mathrm{P}$ at a rate of $60 \mathrm{~kg} \mathrm{fed}^{-1}(1 \mathrm{fed}$ $=4200 \mathrm{~m}^{2}$ ) in clay soils and $80 \mathrm{~kg} \mathrm{fed}^{-1}$ in sandy soils with spraying $\mathrm{Zn}$ at a rate of $250 \mathrm{mg} \mathrm{L}^{-1}$ with the Mycorrhizae and Azotobacter applications. This treatment could achieve 5.45 and $2.21 \mathrm{Mg} \mathrm{fed}^{-1}$ for straw and grains respectively in the sandy soil while, 9.5 and $4.16 \mathrm{Mg}$ $\mathrm{fed}^{-1}$ in the clay soil. This study revealed that combining bio- and mineral $\mathrm{P}$ and $\mathrm{Zn}$ fertilizers application could decrease the negative effect of high $\mathrm{Fe}$ content in soils and increased wheat production.
\end{abstract}

Keywords: New Valley soils, P, Zn, Mycorrhizae, Azotobacter, Iron content, Wheat

Several studies have reported that application of organic and/or bio-fertilizers along with mineral fertilizers increase yield and biochemical components of wheat. For example Tayebeh et al. (2010) found that the highest wheat grain yield was achieved when applied mineral $\mathrm{N}$ fertilizer and compost. Shah et al. (2010) reported that the application of $\mathrm{N}$ fertilizer as urea with $25 \% \mathrm{~N}$ from farm yard manure or poultry manure or city waste achieved optimum wheat yield and reduced fertilizer cost by 50\%. Applications of humic acid with mineral fertilizers achieved highest yield of wheat as well (Tahir et al., 2011). Fadl-Allah et al. (2010) demonstrated that the use of bio-fertilizer with mineral $\mathrm{N}$ fertilizer optimized wheat production and nutrient contents. Singhal et al. (2012) reported that combining the application of mineral $\mathrm{P}$ along with mycorrhizae 
could achieve the highest yield of wheat. Castillo et al. (2012) demonstrated the beneficial effect of mycorrhizae on P uptake in wheat, barley and oats. Hasanpour et al. (2012) found that inoculation of mycorrhizae and/or Azotobacter significantly increased wheat grain yields. In the same respect, Mir et al. (2013) demonstrated that mycorrhiza and Azotobacter applications could improve wheat growth and seed production even under drought stress conditions. Moola et al. (2014) reported that the highest increase in wheat productivity, grain quality and nutrient uptake was recorded with the application of green manure + farmyard manure + bio-fertilizers. Barker and Tagu (2002) evidenced the beneficial effect of mycorrhizae by producing phyto-hormones like auxins and cytokines, which promotes plant growth and consequently the yield.

Soils with high content in Fe retard the uptake of other nutrients, like $\mathrm{P}$ and $\mathrm{Zn}$. For instance, Wandruszka (2006) revealed that high Fe content in soil decreases $\mathrm{P}$ content in the leaf, stem and root of wheat. However, high $\mathrm{P}$ additions could reduce the accumulation of Fe into wheat leaves (Handreck, 2006). Furthermore, Li et al. (2007) reported that higher available soil $\mathrm{P}$ significantly decreased crop micronutrients, possibly because of their precipitation as metal phosphates. Hasina et al. (2011) stated that the growth performance of wheat was highly influenced by the application of two times spray of $0.5 \% \mathrm{~N}, 0.5 \% \mathrm{~K}$ and $0.5 \% \mathrm{Zn}$ solutions. Moghadam et al. (2012) reported that the wheat yield increased with foliar application of $\mathrm{B}$ and $\mathrm{Zn}$. Niemi et al (2014) stated that application of $\mathrm{Fe}$ at high levels decreased the concentrations of $\mathrm{Zn}, \mathrm{Cu}$ and $\mathrm{Mn}$. The New Valley soils are high in Fe content causes severe problems regarding uptake of $\mathrm{P}$ and $\mathrm{Zn}$ by plants. Therefore, the present study was undertaken to study the possibility of improving yield and biochemical components of wheat under the New Valley conditions by integrating mineral $\mathrm{P}$ fertilizers, foliar spray of $\mathrm{Zn}$ and bio-fertilizers in order to overcome the problems caused by high Fe content.

\section{Materials and Methods}

Field experiments in two successive seasons (2012/2013-2013/2014) were carried out in split-split plot design with three replications in two sites at the New Valley, southern Egypt. The first site was at El-Kharaga Oasis (El-Monera villages) and the second was at El-Dakhlla Oasis (Al-Kaser). The first experiment (sandy soil) located at $27^{\circ} 47.7^{\prime} 42^{\prime \prime} \mathrm{N}$ and $30^{\circ} 24.7^{\prime} 56^{\prime \prime} \mathrm{E}$, while the second one (clay soil) located at $28^{\circ} 20.6^{\prime} 24^{\prime \prime} \mathrm{N}$ and $31^{\circ} 59.9^{\prime} 58^{\prime \prime}$ E. The plot area was $90 \mathrm{~m}^{2}$ at Al-Kaser location with flood irrigation system, while was 64 $\mathrm{m}^{2}$ at the El-Monera with sprinkler irrigation system. The used cultivar in each site was Sakha 93, which is popular in these sites. The bio-fertilizer treatments constituted the main plots, $\mathrm{Zn}$ foliar application in sub plots and $\mathrm{P}$ treatments in sub-sub plots.

Table 1 shows the analytical data of soil and water at the experimental sites. A basal dose of $\mathrm{N}$ as ammonium nitrate and $\mathrm{K}$ as potassium sulfate was applied as 70 and $50 \mathrm{~kg} \mathrm{fed}^{-1}$, respectively in sandy soil, where 50 and $25 \mathrm{~kg} \mathrm{fed}^{-1}$ was

Egypt. J. Soil Sci. 56, No. 1 (2016) 
applied in clay soil. The fertilizers of $\mathrm{N}$ and $\mathrm{K}$ were divided to three equal split doses and applied at 20,60, and 90 days after sowing following the general recommendation in the study area. Farm yard manure applied at rate 25 and 15 $\mathrm{m}^{3} \mathrm{fed}^{-1}$ for sandy and clay soils respectively. Four rates of $\mathrm{P}$ as calcium superphosphate and three treatments (without $\mathrm{Bio}_{0}$ and with $\mathrm{Bio}_{1}, \mathrm{Bio}_{2}$ ) of biofertilizers (Microheyzae and Azotobacter) were applied as shown in Table 2. Phosphorus fertilizer was mixed with the soil during seedbed preparation. Foliar $\mathrm{Zn}$ was applied as zinc sulphate at two rates $\left(0,250 \mathrm{mg} \mathrm{L}^{-1}\right)$ at three events during the growing season.

One rate of foliar NPK fertilizers divided to three doses and applied at the same times of $\mathrm{Zn}$ applications for all studied treatments except control as following; first dose ${ }_{1}$ [ $1 \mathrm{~kg}$ of $\left.(20 / 20 / 20)\right] / 200 \mathrm{~L}$ water, second dose $_{2}[0.75 \mathrm{~kg}$ of $(20 / 20 / 20)+0.25 \mathrm{~kg}$ of $(0 / 80 / 0)+0.25 \mathrm{~kg}(0 / 0 / 50)] / 200 \mathrm{~L}$ water and third dose $_{3}$ $[0.5 \mathrm{~kg}$ of $(20 / 20 / 20)+0.35 \mathrm{~kg}$ of $(0 / 80 / 0)+0.4 \mathrm{~kg}(0 / 0 / 50)] / 200 \mathrm{~L}$ water.

TABLE 1. Some physical and chemical properties of the soil and chemical analyses of the irrigation water at the experimental sites.

\begin{tabular}{|c|c|c|c|c|c|c|c|c|c|}
\hline \multirow{2}{*}{$\begin{array}{c}\text { Depth } \\
\text { cm }\end{array}$} & \multirow{2}{*}{$\begin{array}{l}\mathrm{pH} \\
1: 1 \\
\end{array}$} & \multirow{2}{*}{$\begin{array}{c}E C \\
\mathrm{dS} \mathrm{m}^{-1}\end{array}$} & O.M & $\mathrm{CaCO}_{3}$ & Sand & Silt & Clay & \multirow{2}{*}{$\begin{array}{c}\text { CEC } \\
\text { meq/100g }\end{array}$} & \multirow[t]{2}{*}{ Texture } \\
\hline & & & \multicolumn{5}{|c|}{$\%$} & & \\
\hline \multicolumn{10}{|c|}{ Sandy soil } \\
\hline $0-30$ & 7.93 & 0.92 & 0.78 & 1.36 & 87.52 & 8.23 & 4.25 & 3.4 & Sandy \\
\hline $30-60$ & 7.68 & 0.65 & 0.39 & 1.16 & 85.37 & 9.45 & 5.18 & 4.3 & Sandy \\
\hline \multicolumn{10}{|c|}{ Clay soil } \\
\hline $0-30$ & 8.48 & 2.43 & 1.23 & 1.94 & 21.46 & 25.31 & 53.23 & 29.6 & Clayey \\
\hline $30-60$ & 8.25 & 1.56 & 0.83 & 1.76 & 19.51 & 26.37 & 54.12 & 30.7 & Clayey \\
\hline \multicolumn{10}{|c|}{ Soluble cations and anions $\left(\mathrm{meq} \mathrm{L}^{-1}\right)$, amount of total antioxidants and total phenols } \\
\hline \multicolumn{10}{|c|}{ Sandy soil } \\
\hline & $\mathrm{Na}$ & $\mathrm{K}$ & $\mathrm{Ca}$ & $\mathrm{Mg}$ & $\mathrm{HCO}_{3}$ & $\mathrm{Cl}$ & $\mathrm{SO}_{4}$ & T. phenol ${ }^{* *}$ & T.A.A ${ }^{*}$ \\
\hline $0-30$ & 3.42 & 1.22 & 1.98 & 2.58 & 1.85 & 3.99 & 3.36 & 323 & 85 \\
\hline $30-60$ & 2.51 & 0.95 & 1.32 & 1.74 & 1.20 & 3.20 & 2.12 & 186 & 54 \\
\hline & & & & & & Clay & oil & & \\
\hline $0-30$ & 12.7 & 1.67 & 5.20 & 4.74 & 2.32 & 16.36 & 5.62 & 572 & 157 \\
\hline $30-60$ & 8.75 & 0.97 & 3.89 & 1.99 & 1.88 & 9.86 & 3.86 & 395 & 125 \\
\hline & & & & Available $\mathrm{n}$ & ients (mg & & & & \\
\hline & $\mathrm{N}$ & & $\mathrm{P}$ & $\mathrm{K}$ & $\mathrm{Fe}$ & $\mathrm{Mn}$ & & $\mathrm{Zn}$ & $\mathrm{Cu}$ \\
\hline & & & & & y soil & & & & \\
\hline $0-30$ & 16.3 & & .65 & 43 & 17.89 & 6.52 & & 0.25 & 0.12 \\
\hline $30-60$ & 14.2 & & 1.16 & 55 & 19.93 & 8.54 & & 0.28 & 0.13 \\
\hline & & & & & soil & & & & \\
\hline $0-30$ & 39.6 & & 2.93 & 246 & 31.9 & 12.9 & & 0.58 & 0.32 \\
\hline $30-60$ & 32.4 & & 2.25 & 283 & 38.4 & 18.4 & & 0.47 & 0.28 \\
\hline & & & Some c & emical pro & rties of irr & tion wate & & & \\
\hline Soils & $\mathrm{nH}$ & EC & $\mathrm{Na}$ & $\begin{array}{ll}\mathrm{K} \\
\end{array}$ & $\mathrm{Ca}$ & $\mathrm{Mg}$ & $\mathrm{HCO}_{3}$ & $\mathrm{Cl}$ & $\mathrm{SO}_{4}$ \\
\hline SOIIS & рП & $\mathrm{dS} / \mathrm{m}$ & & & & $\mathrm{me} / \mathrm{L}$ & & & \\
\hline Sandy & 7.83 & 0.45 & 1.87 & 0.63 & 0.93 & 1.11 & 0.98 & 3.12 & 0.43 \\
\hline Clay & 8.07 & 0.74 & 2.70 & 0.95 & 1.59 & 2.16 & 1.95 & 4.65 & 0.81 \\
\hline
\end{tabular}


TABLE 2. Treatments of $P$ (as $P_{2} O_{5}$ ), $\mathrm{Zn}$ and bio-fertilizers in wheat at the experimental sites.

\begin{tabular}{|c|c|c|}
\hline \multirow{2}{*}{ Fertilizers } & Sandy soil & Clay soil \\
\cline { 2 - 3 } & \multicolumn{2}{|c|}{ kg fed $^{-\mathbf{1}}$} \\
\hline Control & 0 & 0 \\
\hline $\mathrm{P}_{1}$ & 15 & 20 \\
\hline $\mathrm{P}_{2}$ & 30 & 40 \\
\hline $\mathrm{P}_{3}$ & 45 & 60 \\
\hline $\mathrm{P}_{4}$ & 60 & 80 \\
\hline $\mathrm{Zn}$ & \multicolumn{2}{|c|}{$\mathrm{Zn}\left(0,250 \mathrm{mg} \mathrm{L}^{-1}\right)$ added as foliar spray } \\
\hline Bio-fertilizer & & Mycorrhizae $\left(8 \mathrm{~kg} \mathrm{fed}^{-1}\right)$ added to soil with P \\
\hline Bio-fertilizer & & Mycorrhizae $+($ Azotobacater, $1 \%$ added as foliar spray with $\mathrm{Zn})$ \\
\hline
\end{tabular}

Azotobacter isolate (H-A) was used in the experiments. It has $\mathrm{N}_{2}$-ase activity, $456 \mu \mathrm{CC}_{2} \mathrm{H}_{4} \mathrm{~h}^{-1} \mathrm{l}^{-1}$, phosphate solubilization, $1.54 \mathrm{mg} \mathrm{P} \mathrm{l}^{-1}$, indole-3-acetic acid (IAA) was 0.16 , gibberellic acid (GA3) was 3.2, Cytokinine was $26 \mu \mathrm{g} \mathrm{ml}^{-1}$, enzyme production: Amylase was $(+++)$, Protease was $(+)$, Protease was $(+)$ and Lipase was (+). Fresh liquid of Azotobacter culture of $48 \mathrm{hr}$ old at a rate of $10^{8} \mathrm{CFU} / \mathrm{ml}$ was added as foliar to plants at one rate $(2 \mathrm{~L} / 200 \mathrm{~L})$ with $\mathrm{Zn}$ Foliar application.

For mycorrhiza, spores were isolated from soil pre-inoculated with mycorrhiza (Glomusmacrocarpium) by the wet-sieving and decantation method was used as described by Gerdeman and Nicolson (1963). The mycorrhiza was mixed with pure clay and used to inoculate the experiment plots with one rate $\left(8 \mathrm{~kg} \mathrm{fed}^{-1}\right)$ with seedbed preparation. Phosphate dissolving bacteria, (PDB) were Arbuscular Mycorrhizal Fungi (AMF), Bacillus sp and Pseudomonas fluorescens which achieved maximum yield when applied with the recommended dose of superphosphate (Neetu et al., 2012). The densities of azotobacter in the phyllosphere were determined by cutting $1 \mathrm{~cm}^{2}$ of leaf of each plant and transferred to bottles containing $100 \mathrm{ml}$ sterilized water and shaken vigorously for 15 minutes (Mikhail, 2002) then was analyzed by using modified Ashby's medium. Microbiological analyses of rhizosphere of wheat plants were determined including total microbial counts by plating on Bunt and Rovira agar medium according to Abdel-Hafez (1966). The most probable number (MPN) of azotobacter was determined after incubating the tubes at $28 \pm 2 \mathrm{C}^{\circ}$ for 7 days on modified Ashby's medium ((Hill, 2000). The rates of $\mathrm{CO}_{2}$ evolution were determined according to Page et al. (1984)

Initial Soil samples were collected from two layers $(0-30$ and $30-60 \mathrm{~cm})$ for physical and chemical analysis. Plant samples were collected at physiological maturity. Yield components were determined as biological yield, grain weight, straw weight, weight of 1000 grain, weight of spikes $/ \mathrm{m}^{2}$ and number of spikes $/ \mathrm{m}^{2}$ in both seasons. Soil and plant samples were analyzed for macro and micro

Egypt. J. Soil Sci. 56, No. 1 (2016) 
nutrients according to Cottenie et al. (1982). Soil analyses were accomplished according to Page et al. (1984) and Klute (1986). Total antioxidants measurements and total phenol in soil and wheat plants were determined according to Rimmer (2009). The analysis of variance (ANOVA) was used to determine the effect of treatments on yield parameters. Least significant differences (LSD) test was used to determine the differences between treatments means at 5\% probability level according to Gomez and Gomez (1984).

\section{Results and Discussion}

Effect of the integration between mineral and bio-fertilizers on wheat yield

Data presented in Table 3 showed that yield parameters of wheat were markedly affected by $\mathrm{P}$ and $\mathrm{Zn}$ treatments. Yield parameters of wheat were much higher in the clay soil than in the sandy soil. Increasing the rate of $\mathrm{P}$ and $\mathrm{Zn}$ increased yield parameters compared with other treatments. The best treatment appears to be $\mathrm{P}_{4}+\mathrm{Zn}_{1}$. Bio-fertilizers treatments increased yield parameters of wheat when combined with mineral fertilizers. The most effective treatment for wheat yield was $\left(\mathrm{P}_{4}+\mathrm{Zn}_{1}\right.$ plus Mycorrhizae + Azotobacter $)$ which achieved 5.45 and $2.21 \mathrm{Mg} \mathrm{fed}^{-1}$ for straw and grain, respectively in sandy soil, while 9.5 and $4.16 \mathrm{Mg} \mathrm{fed}^{-1}$ were achieved in clay soil.

Foliar Zn application increased yield about 51.9 and $57.8 \%$ above control treatment for straw and grains respectively in sandy soil, while 52.9 and $49.8 \%$ in clay soil. Yield components of wheat were increased with increasing $\mathrm{P}$ soil applications rates where the highest increases values at $\mathrm{P}_{4}$ (superior treatment) while the lowest increases values was at $\mathrm{P}_{1}$ treatment in both studied soils. Straw and grain of wheat under superior treatment $\left(\mathrm{P}_{4}+\mathrm{Zn}_{1}\right.$ plus Mycorrhizae +Azotobacter) were higher than control about 56.3 and $63 \%$ for straw and grains respectively in sandy soil while, 55.2 and $52.4 \%$ in the clay soil.

Mycorrhizae had higher increase in grains yield than Azotobacter, while Azotobacter had higher straw yield than Mycorrhizae. Combining both biofertilizers sources had higher significantly values of grains and straw yield than the individual sources.

These results could be due to that application of mineral and bio-fertilizers increasing available nutrients contents in the soil and consequently enhancing plant growth and yield. Mycorrhizae application increased available $\mathrm{P}$ in soil because it dissolves soil $\mathrm{P}$, while Azotobacter application increased available $\mathrm{N}$ in soil since it is fixing $\mathrm{N}$ from the air. Moreover, bio-fertilizers produce hormones like auxins and cytokines which regulating plant growth and increase plant productivity. These facts about bio-fertilizers are barreled with Barker and Tagu ( 2002); Castillo et al. (2012); Amanullah et al. (2012); Kowsar et al., (2014); and Garshasbi et al. (2014), where mineral fertilizers effect agreed with Hasina et al.,(2011); Moghadam et al.,(2012); Abbas et al. (2013); and Ehsan et al. (2014) 
TABLE 3 . Effect of mineral and bio-fertilizers on wheat yield parameters in the studied soil.

\begin{tabular}{|c|c|c|c|c|c|c|c|c|c|c|}
\hline \multirow{3}{*}{\multicolumn{3}{|c|}{ Treatments }} & 焉 & 节 & \multirow{2}{*}{ 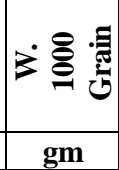 } & \multirow{3}{*}{$\frac{3}{\mathrm{~kg} / \mathrm{m}^{2}}$} & 苞 & 芜 & \multirow{2}{*}{ 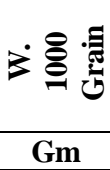 } & \multirow{2}{*}{$\frac{\mathrm{kg} / \mathrm{m}^{2}}{\tilde{n}^{2}}$} \\
\hline & & & \multicolumn{2}{|c|}{ Ton/fed } & & & \multicolumn{2}{|c|}{ Ton/fed } & & \\
\hline & & & \multicolumn{3}{|c|}{ Sandy soil } & & \multicolumn{4}{|c|}{$\begin{array}{l}\text { Clay soil } \\
\end{array}$} \\
\hline \multicolumn{11}{|c|}{ Mineral fertilizer } \\
\hline \multirow{9}{*}{$\stackrel{\circ}{\circ}$} & \multirow{5}{*}{ งี } & $\begin{array}{c}\text { Contr } \\
\text { ol }\end{array}$ & 2.21 & 0.76 & 23.9 & 0.39 & 3.80 & 1.80 & 33.8 & 0.93 \\
\hline & & $\mathrm{P}_{1}$ & 3.70 & 1.33 & 40.6 & 0.62 & 6.65 & 3.16 & 57.2 & 1.50 \\
\hline & & $\mathrm{P}_{2}$ & 4.18 & 1.58 & 48.2 & 0.71 & 6.98 & 3.26 & 61.5 & 1.57 \\
\hline & & $\mathrm{P}_{3}$ & 4.47 & 1.77 & 51.9 & 0.86 & 7.41 & 3.52 & 66.5 & 1.63 \\
\hline & & $\mathrm{P}_{4}$ & 4.80 & 1.95 & 56.5 & 0.94 & 7.75 & 3.59 & 70.9 & 1.73 \\
\hline & \multirow{4}{*}{ సี } & $\mathrm{P}_{1}$ & 3.92 & 1.42 & 43.7 & 0.66 & 6.73 & 3.23 & 62.9 & 1.55 \\
\hline & & $\mathrm{P}_{2}$ & 4.30 & 1.67 & 49.8 & 0.76 & 7.34 & 3.29 & 63.4 & 1.63 \\
\hline & & $\mathrm{P}_{3}$ & 4.57 & 1.87 & 53.6 & 0.88 & 7.64 & 3.71 & 69.2 & 1.69 \\
\hline & & $\mathrm{P}_{4}$ & 4.81 & 1.99 & 60.5 & 1.04 & 8.22 & 3.77 & 73.0 & 1.77 \\
\hline \multicolumn{11}{|c|}{ Bio-fertilizers (Mycorrhizae) } \\
\hline \multirow{9}{*}{$\stackrel{\circ}{0}$} & \multirow{5}{*}{ 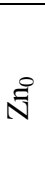 } & Control & 2.24 & 0.78 & 25.1 & 0.41 & 3.83 & 1.86 & 35.4 & 0.99 \\
\hline & & $\mathrm{P}_{1}$ & 3.81 & 1.37 & 42.7 & 0.66 & 6.70 & 3.25 & 60.1 & 1.56 \\
\hline & & $\mathrm{P}_{2}$ & 4.35 & 1.64 & 51.1 & 0.77 & 7.08 & 3.39 & 65.2 & 1.61 \\
\hline & & $\mathrm{P}_{3}$ & 4.65 & 1.88 & 54.5 & 0.89 & 7.90 & 3.66 & 69.8 & 1.73 \\
\hline & & $\mathrm{P}_{4}$ & 5.04 & 2.06 & 60.5 & 0.98 & 7.92 & 3.72 & 72.6 & 1.75 \\
\hline & \multirow{4}{*}{$\overline{\mathrm{N}}$} & $\mathrm{P}_{1}$ & 4.03 & 1.47 & 45.9 & 0.69 & 6.86 & 3.33 & 66.0 & 1.60 \\
\hline & & $\mathrm{P}_{2}$ & 4.47 & 1.74 & 52.7 & 0.81 & 7.98 & 3.41 & 67.2 & 1.68 \\
\hline & & $\mathrm{P}_{3}$ & 4.75 & 1.94 & 56.3 & 0.94 & 8.56 & 3.85 & 72.7 & 1.79 \\
\hline & & $\mathrm{P}_{4}$ & 5.05 & 2.11 & 64.7 & 1.09 & 8.94 & 3.96 & 75.3 & 1.81 \\
\hline \multicolumn{11}{|c|}{ Bio-fertilizers (Mycorrhizae+ Azotobacter ) } \\
\hline \multirow{9}{*}{$\stackrel{\delta}{\infty}$} & \multirow{5}{*}{ సี } & $\begin{array}{c}\text { Contr } \\
\text { ol }\end{array}$ & 2.28 & 0.80 & 26.4 & 0.43 & 4.15 & 1.91 & 40.0 & 1.03 \\
\hline & & $\mathrm{P}_{1}$ & 4.04 & 1.41 & 44.7 & 0.70 & 7.30 & 3.31 & 63.1 & 1.62 \\
\hline & & $\mathrm{P}_{2}$ & 4.64 & 1.70 & 55.1 & 0.82 & 7.92 & 3.52 & 69.1 & 1.68 \\
\hline & & $\mathrm{P}_{3}$ & 4.93 & 1.95 & 58.1 & 0.94 & 8.67 & 3.81 & 73.2 & 1.79 \\
\hline & & $\mathrm{P}_{4}$ & 5.39 & 2.15 & 64.7 & 1.04 & 8.77 & 3.86 & 77.6 & 1.81 \\
\hline & \multirow{4}{*}{ งี } & $\mathrm{P}_{1}$ & 4.28 & 1.50 & 50.0 & 0.75 & 7.45 & 3.40 & 69.3 & 1.67 \\
\hline & & $\mathrm{P}_{2}$ & 4.79 & 1.87 & 57.7 & 0.86 & 8.68 & 3.55 & 71.2 & 1.78 \\
\hline & & $\mathrm{P}_{3}$ & 5.04 & 2.03 & 61.7 & 0.99 & 9.36 & 4.06 & 76.3 & 1.91 \\
\hline & & $\mathrm{P}_{4}$ & 5.45 & 2.21 & 69.2 & 1.13 & 9.50 & 4.16 & 78.6 & 1.93 \\
\hline \multicolumn{3}{|c|}{ LSD $_{0.05}$ Bio } & 0.082 & 0.026 & 1.23 & 0.017 & 0.20 & 0.048 & 1.35 & 0.03 \\
\hline \multicolumn{3}{|c|}{$\mathrm{LSD}_{0.05} \mathrm{Zn}$} & 0.007 & 0.005 & 0.19 & 0.003 & 0.03 & 0.008 & 0.18 & 0.01 \\
\hline \multicolumn{3}{|c|}{$\mathrm{LSD}_{0.05} \mathrm{P}$} & 0.059 & 0.027 & 0.76 & 0.013 & 0.10 & 0.042 & 0.79 & 0.02 \\
\hline LSI & 0.05 & Biox Zn & 0.010 & 0.007 & 0.27 & 0.003 & 0.04 & 0.011 & 0.16 & 0.01 \\
\hline $\mathrm{LS}$ & $D_{0.0}$ & Bio $\times \mathrm{P}$ & 0.084 & 0.038 & 1.08 & 0.018 & 0.14 & 0.059 & 1.02 & 0.02 \\
\hline & 500 & $\mathrm{Zn} \times \mathrm{P}$ & 0.103 & 0.046 & 1.32 & 0.022 & 0.17 & 0.073 & 1.37 & 0.03 \\
\hline$\overline{\mathrm{LSI}}$ & 005 & 3 factors & 0.109 & 0.049 & 1.39 & 0.023 & 0.18 & 0.077 & 1.45 & 0.03 \\
\hline
\end{tabular}

$$
\text { W: Weight }
$$

Egypt. J. Soil Sci. 56, No. 1 (2016) 
Effect of the integration between mineral and bio-fertilizers on nutrients uptake by wheat

Regarding the effect of mineral $\mathrm{P}$ and $\mathrm{Zn}$ on nutrients uptake, Tables 4 and 5 showed that application of $\mathrm{P}$ and $\mathrm{Zn}$ increased $\mathrm{N}, \mathrm{P}, \mathrm{K}, \mathrm{Zn}$ and $\mathrm{Cu}$ uptake at harvest. On the other hand, Fe and Mn uptake by wheat have decreased. These results could be due to the antagonistic effect between the absorbed Fe and $\mathrm{P}$.

TABLE 4 . Effect of mineral and bio-fertilizers on wheat nutrients uptake at the first site (sandy soil).

\begin{tabular}{|c|c|c|c|c|c|c|c|c|c|c|c|c|c|c|c|}
\hline \multirow{3}{*}{\multicolumn{2}{|c|}{ Treatments }} & \multicolumn{7}{|c|}{ Nutrients uptake of straw } & \multicolumn{7}{|c|}{ Nutrients uptake of grain } \\
\hline & & $\mathbf{N}$ & $\mathbf{P}$ & \begin{tabular}{|l|}
$\mathbf{K}$ \\
\end{tabular} & $\mathbf{F e}$ & Mn & $\mathbf{Z n}$ & $\mathrm{Cu}$ & $\mathbf{N}$ & $\mathbf{P}$ & $\mathbf{K}$ & $\mathbf{F e}$ & Mn & $\mathbf{Z n}$ & $\overline{\mathrm{Cu}}$ \\
\hline & & \multicolumn{3}{|c|}{ Kg/fed } & \multicolumn{4}{|c|}{ g/fed } & \multicolumn{3}{|c|}{ Kg/fed } & \multicolumn{4}{|c|}{ g/fed } \\
\hline \multicolumn{16}{|c|}{ Mineral fertilizers } \\
\hline & $\begin{array}{c}\text { Contr } \\
\text { ol }\end{array}$ & 5.3 & 1.8 & 4.6 & 690 & 365 & 5 & 3.5 & 2.2 & 1.1 & 1.3 & 271 & 140 & 3 & 1.4 \\
\hline & $\mathrm{P}_{1}$ & 13.7 & 5.6 & 15.5 & 1062 & 570 & 13 & 6.1 & 5.9 & 2.5 & 3.7 & 464 & 229 & 8 & 2.5 \\
\hline & $\mathrm{P}_{2}$ & 17.1 & 10.0 & 18.0 & 1137 & 619 & 16 & 7.1 & 7.6 & 4.4 & 4.9 & 529 & 254 & 10 & 3.1 \\
\hline & $\mathrm{P}_{3}$ & 19.2 & 13.9 & 21.0 & 1140 & 621 & 21 & 7.8 & 9.0 & 6.4 & 6.0 & 582 & 271 & 13 & 3.5 \\
\hline & $\overline{\mathrm{P}_{4}}$ & 22.6 & 17.3 & 24.0 & 1133 & 610 & 25 & 8.5 & 11.1 & 8.0 & 7.0 & 608 & 277 & 15 & 4.0 \\
\hline & $\mathrm{P}_{1}$ & 16.1 & 6.3 & 16.9 & 1031 & 517 & 251 & 7.6 & 6.7 & 3.1 & 4.4 & 447 & 223 & 118 & 3.0 \\
\hline & $\mathrm{P}_{2}$ & 19.8 & 10.8 & 20.6 & 1079 & 520 & 297 & 9.4 & 9.0 & 5.3 & 6.0 & 506 & 247 & 141 & 3.7 \\
\hline & $\mathrm{P}_{3}$ & 22.9 & 15.1 & 24.2 & 1074 & 512 & 340 & 10.1 & 10.8 & 7.3 & 7.3 & 552 & 247 & 163 & 4.4 \\
\hline & $\mathrm{P}_{4}$ & 25.0 & 17.8 & 26.5 & 1029 & 495 & 370 & 11.0 & \begin{tabular}{|l|}
11.9 \\
\end{tabular} & 8.6 & 8.4 & 571 & 251 & 177 & 4.9 \\
\hline \multicolumn{16}{|c|}{ Bio-fertilizers (Mycorrhizae) } \\
\hline \multirow{9}{*}{.} & $\begin{array}{c}\text { Contr } \\
\text { ol }\end{array}$ & 6.0 & 3.1 & 4.9 & 629 & 340 & 7 & 3.6 & 2.5 & 2.0 & 1.4 & 266 & 137 & 3 & 1.5 \\
\hline & $\mathrm{P}_{1}$ & 14.9 & 10.7 & 16.4 & 964 & 518 & 14 & 6.4 & 6.3 & 4.7 & 4.0 & 455 & 230 & 9 & 2.8 \\
\hline & $\mathrm{P}_{2}$ & 18.7 & 15.7 & 19.1 & 1070 & 561 & 17 & 7.6 & 8.2 & 6.4 & 5.7 & 533 & 254 & 11 & 3.5 \\
\hline & $\mathrm{P}_{3}$ & 20.9 & 20.9 & 22.8 & 1102 & 563 & 23 & 8.3 & 10.0 & 9.2 & 7.3 & 594 & 278 & 15 & 4.1 \\
\hline & $\mathrm{P}_{4}$ & 24.7 & 26.7 & 26.2 & 1094 & 570 & 29 & 9.1 & 12.2 & 11.3 & 8.9 & 622 & 286 & 18 & 4.6 \\
\hline & $P_{1}$ & 17.7 & 12.5 & 17.7 & 923 & 463 & 284 & 8.3 & \begin{tabular}{|l|}
7.5 \\
\end{tabular} & \begin{tabular}{|l|l|}
5.4 \\
\end{tabular} & 4.9 & 467 & 219 & 125 & 3.4 \\
\hline & $\mathrm{P}_{2}$ & 21.0 & 17.4 & 21.9 & 961 & 487 & 355 & 10.1 & 9.4 & 7.5 & 7.0 & 529 & 251 & 151 & 4.2 \\
\hline & $\mathrm{P}_{3}$ & 23.3 & \begin{tabular}{|l}
22.8 \\
\end{tabular} & 25.2 & 988 & 466 & 399 & 10.9 & \begin{tabular}{|l|}
11.3 \\
\end{tabular} & \begin{tabular}{|l|}
10.1 \\
\end{tabular} & 8.5 & 568 & 246 & 174 & 4.8 \\
\hline & $\mathrm{P}_{4}$ & 27.3 & 27.8 & 31.3 & 990 & 460 & 447 & 11.9 & \begin{tabular}{|l|}
13.3 \\
\end{tabular} & 12.0 & \begin{tabular}{|l|}
10.1 \\
\end{tabular} & 584 & 260 & 192 & 5.3 \\
\hline \multicolumn{16}{|c|}{ Bio-fertilizers (Mycorrhizae+ Azotobacter ) } \\
\hline & Control & 6.6 & 3.6 & 5.7 & 613 & 340 & 7 & 3.8 & 2.8 & 2.3 & 1.8 & 266 & 137 & 4 & 1.7 \\
\hline & $\mathrm{P}_{1}$ & 21.4 & 11.7 & 18.2 & 1002 & 533 & 15 & 6.9 & 8.9 & 5.2 & 4.4 & 458 & 219 & 10 & 3.0 \\
\hline & $\mathrm{P}_{2}$ & 26.0 & 17.6 & 22.7 & 1086 & 561 & 20 & 8.3 & 11.2 & 7.3 & 6.6 & 530 & 241 & 12 & 3.7 \\
\hline & $\mathrm{P}_{3}$ & 30.6 & 23.7 & 27.1 & 1090 & 562 & 25 & 9.1 & 14.0 & 9.9 & 9.0 & 595 & 257 & 16 & 4.3 \\
\hline & $\mathrm{P}_{4}$ & 36.1 & 29.6 & 32.3 & 1137 & 577 & 32 & 10.1 & \begin{tabular}{|l|}
16.6 \\
\end{tabular} & 12.0 & 10.5 & 630 & 258 & 20 & 4.9 \\
\hline & $\mathrm{P}_{1}$ & 24.0 & 14.6 & 21.0 & 1010 & 479 & 319 & 9.0 & $\begin{array}{ll}9.8 \\
\end{array}$ & \begin{tabular}{|l|l|}
5.7 \\
\end{tabular} & 5.3 & 453 & 222 & 131 & 3.5 \\
\hline & $\overline{\mathrm{P}_{2}}$ & 29.2 & 20.6 & 24.9 & 1073 & 508 & 378 & 11.0 & \begin{tabular}{|l|}
13.1 \\
\end{tabular} & 8.4 & 8.0 & 540 & 252 & 166 & 4.5 \\
\hline & $\mathrm{P}_{3}$ & 33.3 & 24.7 & 29.7 & 1068 & 474 & 428 & 11.9 & 15.2 & 11.2 & 9.9 & 552 & 250 & 187 & 5.2 \\
\hline & $\mathrm{P}_{4}$ & 38.7 & 31.1 & 35.4 & 1046 & 480 & 488 & 13.2 & \begin{tabular}{|l|}
18.3 \\
\end{tabular} & 13.0 & 11.7 & 586 & 256 & 207 & 5.7 \\
\hline \multicolumn{2}{|c|}{$\mathrm{LSD}_{0.05} \mathrm{Bio}$} & 1.70 & 1.55 & 0.89 & 13 & 8.3 & 7.1 & 0.24 & 0.71 & 0.58 & 0.35 & 1.7 & 1.3 & 1.9 & 0.12 \\
\hline \multirow{2}{*}{\multicolumn{2}{|c|}{\begin{tabular}{|l|} 
LSD $_{0.05} \mathrm{Zn}$ \\
$\mathrm{LSD}_{0.05} \mathrm{P}$
\end{tabular}}} & 0.14 & 0.08 & 0.14 & 4 & 4.5 & 18.3 & 0.13 & 0.07 & 0.05 & 0.06 & 1.1 & 0.7 & 7.8 & 0.04 \\
\hline & & 0.43 & 0.42 & 0.44 & 9 & 4.1 & 5.7 & 0.13 & \begin{tabular}{|l|}
0.21 \\
\end{tabular} & 0.17 & 0.15 & 6.4 & 2.5 & 2.5 & 0.06 \\
\hline \multicolumn{2}{|c|}{$\mathrm{LSD}_{006}$ Biox Zn } & 0.12 & 0.12 & 0.20 & 5 & 6.3 & 25.8 & 0.18 & 0.10 & 0.06 & 0.05 & 1.6 & 1.1 & 6.8 & 0.03 \\
\hline \multicolumn{2}{|c|}{$\mathrm{LSD}_{006} \mathrm{Biox} \mathrm{P}$} & 0.61 & 0.59 & 0.63 & 13 & 5.7 & 8.1 & 0.19 & 0.30 & 0.24 & 0.21 & 8.2 & 3.5 & 3.6 & 0.09 \\
\hline \multicolumn{2}{|c|}{$\mathrm{LSD}_{006} \mathrm{ZnxP}$} & 0.75 & 0.73 & 0.77 & 16 & 7.0 & 9.9 & 0.23 & \begin{tabular}{|l|}
0.37 \\
\end{tabular} & 0.30 & 0.26 & 11.0 & 4.3 & 4.4 & 0.11 \\
\hline \multicolumn{2}{|c|}{$\mathrm{LSD}_{0.06} 3$ factors } & 0.79 & 0.77 & 1.09 & 22 & 9.9 & 14.0 & 0.24 & \begin{tabular}{|l|}
0.39 \\
\end{tabular} & 0.32 & 0.28 & 15.6 & 6.1 & 6.2 & 0.12 \\
\hline
\end{tabular}


TABLE 5 . Effect of mineral and bio-fertilizers on wheat nutrients uptake at the second site (clay soil).

\begin{tabular}{|c|c|c|c|c|c|c|c|c|c|c|c|c|c|c|c|c|}
\hline \multirow{3}{*}{\multicolumn{3}{|c|}{ Treatments }} & \multicolumn{7}{|c|}{ Nutrients uptake of straw } & \multicolumn{7}{|c|}{ Nutrients uptake of grain } \\
\hline & & & $\mathbf{N}$ & $\mathbf{P}$ & $\mathbf{K}$ & $\mathbf{F e}$ & Mn & $\overline{Z n}$ & $\mathbf{C u}$ & $\mathbf{N}$ & $\mathbf{P}$ & $\mathbf{K}$ & $\mathbf{F e}$ & Mn & $\overline{Z n}$ & $\mathbf{C u}$ \\
\hline & & & \multicolumn{3}{|c|}{ Kg/fed } & \multicolumn{4}{|c|}{ g/fed } & \multicolumn{3}{|c|}{ Kg/fed } & \multicolumn{4}{|c|}{ g/fed } \\
\hline \multicolumn{17}{|c|}{ Mineral fertilizers } \\
\hline \multirow{9}{*}{$\stackrel{0}{0}$} & & Control & 16.0 & 4.6 & 13.3 & 1577 & 745 & 29 & 11.7 & 9.4 & 3.1 & 5.8 & 922 & 392 & 19 & 6.8 \\
\hline & & $\mathrm{P}_{1}$ & 50.5 & 14.6 & 39.2 & 2687 & 1210 & 83 & 21.0 & 28.8 & 9.8 & 16.7 & 1558 & 648 & 55 & 12.5 \\
\hline & & $\mathrm{P}_{2}$ & 54.4 & 21.6 & 42.6 & 2701 & 1208 & 96 & 23.4 & 30.0 & 13.7 & 17.9 & 1549 & 626 & 59 & 14.1 \\
\hline & & $\mathrm{P}_{3}$ & 60.0 & 31.9 & 50.4 & 2705 & 1200 & 108 & 26.4 & 33.4 & 18.7 & 21.8 & 1602 & 644 & 69 & 16.2 \\
\hline & & $\mathrm{P}_{4}$ & 65.1 & 39.5 & 56.6 & 2658 & 1163 & 119 & 28.9 & 34.5 & 23.3 & 23.7 & 1562 & 628 & 74 & 17.0 \\
\hline & & $\mathrm{P}_{1}$ & 53.2 & 16.2 & 42.4 & 2598 & 1184 & 595 & 30.1 & 31.3 & 11.3 & 18.4 & 1563 & 610 & 358 & 17.6 \\
\hline & & $\mathrm{P}_{2}$ & 60.2 & 26.4 & 47.7 & 2708 & 1204 & 687 & 33.7 & 34.5 & 16.1 & 19.4 & 1530 & 569 & 374 & 18.5 \\
\hline & & $\mathrm{P}_{3}$ & 64.9 & 34.4 & 55.8 & 2705 & 1169 & 746 & 35.8 & 40.4 & 21.5 & 24.5 & 1651 & 612 & 444 & 21.3 \\
\hline & & $\mathrm{P}_{4}$ & 71.5 & 44.4 & 61.7 & 2770 & 1159 & 832 & 38.9 & 43.0 & 26.0 & 25.6 & 1576 & 573 & 466 & 22.5 \\
\hline \multicolumn{17}{|c|}{ Bio-fertilizers (Mycorrhizae) } \\
\hline \multirow{9}{*}{$\stackrel{\overline{0}}{0}$} & & Control & 17.6 & 8.0 & 13.8 & 808 & 372 & 31 & 12.1 & 10.6 & 4.5 & 6.3 & 487 & 214 & 21 & 7.3 \\
\hline & & $\mathrm{P}_{1}$ & 52.3 & 22.8 & 41.5 & 2573 & 1159 & 88 & 21.7 & 30.6 & 12.4 & 18.2 & 1521 & 634 & 59 & 13.8 \\
\hline & & $\mathrm{P}_{2}$ & 55.9 & 30.4 & 47.4 & 2577 & 1154 & 104 & 24.0 & 32.5 & 16.6 & 20.7 & 1515 & 610 & 65 & 15.2 \\
\hline & & $\mathrm{P}_{3}$ & 65.6 & 42.7 & 60.0 & 2678 & 1193 & 125 & 28.3 & 36.2 & 21.2 & 25.3 & 1548 & 622 & 73 & 17.3 \\
\hline & & $\mathrm{P}_{4}$ & 67.3 & 48.3 & 64.2 & 2503 & 1093 & 133 & 30.0 & 38.3 & 26.0 & 27.2 & 1488 & 599 & 80 & 18.5 \\
\hline & & $\mathrm{P}_{1}$ & 56.3 & 24.7 & 45.3 & 2518 & 1146 & 656 & 30.7 & 33.0 & 14.0 & 20.0 & 1532 & 599 & 384 & 18.6 \\
\hline & & $\mathrm{P}_{2}$ & 67.0 & 37.5 & 57.5 & 2769 & 1229 & 776 & 36.8 & 36.8 & 18.4 & 22.2 & 1490 & 556 & 404 & 19.5 \\
\hline & & $\mathrm{P}_{3}$ & 74.5 & 48.8 & 69.3 & 2816 & 1216 & 870 & 40.9 & 43.9 & 23.9 & 28.1 & 1594 & 589 & 467 & 22.9 \\
\hline & & $\mathrm{P}_{4}$ & 79.6 & 57.2 & 75.1 & 2771 & 1162 & 948 & 43.2 & 46.7 & 28.9 & 30.1 & 1525 & 554 & 497 & 24.3 \\
\hline \multicolumn{17}{|c|}{ Bio-fertilizers (Mycorrhizae+ Azotobacter) } \\
\hline \multirow{9}{*}{$\frac{\delta}{\infty}$} & \multirow{9}{*}{$\mathbf{N}$} & Control & 22.4 & 10.4 & 16.2 & 834 & 382 & 41 & 13.3 & 16.4 & 5.0 & 6.9 & 476 & 208 & 23 & 8.0 \\
\hline & & $\mathrm{P}_{1}$ & 71.5 & 27.0 & 47.5 & 2665 & 1197 & 108 & 24.0 & 47.7 & 13.6 & 19.2 & 1473 & 612 & 64 & 14.5 \\
\hline & & $\mathrm{P}_{2}$ & 93.5 & 34.8 & 58.6 & 2709 & 1212 & 124 & 27.2 & 52.1 & 18.3 & 23.6 & 1478 & 598 & 72 & 15.9 \\
\hline & & $\mathrm{P}_{3}$ & 107.5 & 51.2 & 72.8 & 2740 & 1214 & 139 & 31.6 & 58.3 & 24.4 & 29.0 & 1501 & 602 & 82 & 18.7 \\
\hline & & $\mathrm{P}_{4}$ & 112.3 & 55.3 & 78.9 & 2543 & 1114 & 153 & 33.7 & 60.6 & 28.2 & 31.3 & 1420 & 571 & 86 & 19.7 \\
\hline & & $\mathrm{P}_{1}$ & 82.7 & 29.1 & 51.4 & 2593 & 1185 & 736 & 33.9 & 50.3 & 15.3 & 21.1 & 1486 & 581 & 394 & 19.6 \\
\hline & & $\mathrm{P}_{2}$ & 105.0 & 39.9 & 68.6 & 2830 & 1259 & 878 & 40.6 & 54.7 & 19.9 & 25.6 & 1459 & 543 & 439 & 20.8 \\
\hline & & $\mathrm{P}_{3}$ & 118.9 & 57.1 & 84.2 & 2864 & 1236 & 996 & 45.4 & 64.6 & 26.4 & 32.9 & 1563 & 581 & 515 & 24.8 \\
\hline & & $\mathrm{P}_{4}$ & 125.4 & 62.7 & 88.4 & 2708 & 1131 & 1053 & 46.8 & 67.8 & 31.2 & 34.9 & 1473 & 562 & 549 & 25.9 \\
\hline \multicolumn{3}{|c|}{$\mathrm{LSD}_{0.05}$ Bio } & 7.06 & 2.62 & 2.97 & 36 & 17 & 17 & 3.91 & 0.75 & 0.95 & 0.90 & 31 & 12 & 6 & 0.42 \\
\hline \multicolumn{3}{|c|}{$\mathrm{LSD}_{0.05} \mathrm{Zn}$} & 0.48 & 0.26 & 0.40 & 5 & 1 & 37 & 0.28 & 0.12 & 0.13 & 0.61 & 1 & 2 & 20 & 0.28 \\
\hline \multicolumn{3}{|c|}{$\mathrm{LSD}_{0.05} \mathrm{P}$} & 1.37 & 0.84 & 1.08 & 35 & 15 & 12 & 0.71 & 0.43 & 0.43 & 0.48 & 20 & 7 & 7 & 0.27 \\
\hline \multicolumn{3}{|c|}{$\begin{array}{l}\operatorname{LSD}_{0.05} \text { Biox } \\
\mathrm{Zn}\end{array}$} & 0.68 & 0.37 & 0.56 & 7 & 2 & 53 & 0.40 & 0.10 & 0.18 & 0.86 & 1 & 3 & 17 & 0.24 \\
\hline \multicolumn{3}{|c|}{$\mathrm{LSD}_{0.05}$ Biox P } & 1.93 & 1.18 & 1.53 & 50 & 21 & 18 & 1.01 & 0.61 & 0.61 & 0.68 & 28 & 10 & 9 & 0.38 \\
\hline \multicolumn{3}{|c|}{$\mathrm{LSD}_{0.05} \mathrm{Zn} \times \mathrm{P}$} & 2.37 & 1.45 & 1.87 & 61 & 26 & 22 & 1.24 & 0.74 & 0.74 & 0.83 & 34 & 12 & 11 & 0.47 \\
\hline \multicolumn{3}{|c|}{$\mathrm{LSD}_{0 \mathrm{0}} 3$ factors } & 2.50 & 1.53 & 1.98 & 65 & 28 & 30 & 1.31 & 0.78 & 0.78 & 1.18 & 36 & 13 & 16 & 0.49 \\
\hline
\end{tabular}

The soil application of Mycorrhizae increased $\mathrm{P}$ uptake by straw and grain, while foliar application of Azotobacter increased $\mathrm{N}$ uptake by straw and grain. The application of bio-fertilizers (from both sources) with mineral $\mathrm{P}$ and $\mathrm{Zn}$ deceased Fe and Mn uptake.

These results could be due to the role of Mycorrhizae in dissolving $\mathrm{P}$ in soil and Azotobacter in fixing $\mathrm{N}$ from the air. The superior treatment was application of $\mathrm{P}_{4}+\mathrm{Zn}_{1}$ along with Mycorrhizae + Azotobacter compared with the other treatments at Egypt. J. Soil Sci. 56, No. 1 (2016) 
both sites. These results demonstrate that high Fe content and its negative effect on plant growth and yield could be decreased by mineral $\mathrm{P}$ and $\mathrm{Zn}$. This is in agreement with the results obtained by Ewa \& Jolanta (2005); Kefyalew et al. (2006); Wandruszka (2006); Li et al. (2007); Yassen et al. (2010); and Niemi et al. (2014).

The sandy soil had higher response to mineral and bio-fertilizers application than the clay soil. That is logically because of the lower nutrient contents in sandy soils than clay soil, these are facts agreed with obtained by Abbas et al., (2012), Abbas et al. (2011) and Nadim et al. (2011)

Effect of the integration between mineral and bio-fertilizers on biochemical components in wheat

Total phenols and antioxidants contents in straw and grain increased with increasing mineral $\mathrm{P}$ and $\mathrm{Zn}$ application in the two studied soils. Combining application of mineral and bio-fertilizers further increased total phenols and antioxidants contents in straw and grains of wheat (Table 6). These components contents in straw were higher than that in grains. These results agreeable with that obtained with Li et al. (2013).

The bio-fertilizer (Mycorrhizae + Azotobacter) increased total phenols and total antioxidants contents in straw and grains. The superior treatment was $\mathrm{P}_{4}+$ $\mathrm{Zn}_{1}$ combined with application of Mycorrhizae + Azotobacter compared with the other treatments in both soil types. These result agreed with that obtained by Yoshie et al. (2008) and Lachman et al. (2011).

Regarding the effect of soil type, sandy soil had higher response to mineral ( $\mathrm{P}$ $+\mathrm{Zn}$ ) and bio-fertilizers than clayey soil in respect of biochemical. However, the clay soil achieved the highest contents. This is agreed with Ríos et al. (2009); Vaher et al. (2010); and Li et al. (2013).

Effect of the integration between mineral and bio-fertilizers on microbial densities and $\mathrm{CO}_{2}$ evolution

Microbial densities

Generally, the microbial densities in rhizosphere at vegetation stage in all treatments were significantly higher than control as shown in Table 7. The highest counts in rhizosphere were obtained from plants treated with $\mathrm{P}_{4}+\mathrm{Zn}_{1}$ and Mycorrhizae + Azotobacter recording mean values of 92 and $965 \times 10^{2} \mathrm{cfu} \mathrm{g}^{-1}$ dry soil in sandy and clay soils, respectively. The counts declined toward harvesting stage which could be attributed to the unfavorable moisture content of soil due to soil dryness.

The maximum phosphate dissolving bacterial (PDB) counts of wheat rhizosphere was 12.9 and $59 \times 10^{2} \mathrm{CFU} / \mathrm{g}$ D.S) for sandy and clay soils respectively under superior treatment conditions. This result agreed with the obtained by Singhal et al. (2012), Castillo et al. (2012) and Hasanpour et al. (2012) who indicated that the combing the application of mineral $\mathrm{P}$ along with mycorrhiza could achieve the highest yield and $\mathrm{P}$ uptake by wheat.

Egypt. J. Soil Sci. 56, No. 1(2016) 
TABLE 6. Effect of mineral and bio-fertilizers treatments on biochemical components of wheat in studied soils.

\begin{tabular}{|c|c|c|c|c|c|c|c|c|c|}
\hline \multirow{4}{*}{\multicolumn{2}{|c|}{ Treatments }} & \multicolumn{4}{|c|}{$\begin{array}{l}\text { Sandy soil } \\
\end{array}$} & \multicolumn{4}{|c|}{ Clay soil } \\
\hline & & \multirow{2}{*}{\multicolumn{2}{|c|}{$\begin{array}{c}{ }^{1} \mathrm{~T} \text {. antioxidants } \\
\mu \mathrm{g}^{*} \mathrm{Asc} / \mathrm{ml}\end{array}$}} & \multirow{2}{*}{\multicolumn{2}{|c|}{$\begin{array}{c}\text { T. phenols } \\
\text { mol }^{* *} \mathrm{Gal} / \mathrm{ml}\end{array}$}} & \multirow{2}{*}{\multicolumn{2}{|c|}{$\begin{array}{c}\text { T. antioxidants } \\
\mu \mathrm{g} \text { Asc } / \mathrm{ml}\end{array}$}} & \multirow{2}{*}{\multicolumn{2}{|c|}{$\begin{array}{c}\text { T. phenols } \\
\mu \mathrm{mol} \text { Gal } / \mathrm{ml}\end{array}$}} \\
\hline & & & & & & & & & \\
\hline & & Grains & Straw & Grains & Straw & Grains & Straw & Grains & Straw \\
\hline \multicolumn{10}{|c|}{ Mineral fertilizers } \\
\hline & $\begin{array}{c}\text { Contro } \\
1\end{array}$ & 38 & 52 & 119 & 176 & 72 & 93 & 200 & 294 \\
\hline & $\mathrm{P}_{1}$ & 53 & 73 & 158 & 245 & 107 & 137 & 298 & 439 \\
\hline Nี & $\mathrm{P}_{2}$ & 56 & 77 & 165 & 259 & 113 & 145 & 313 & 461 \\
\hline$\circ$ & $\mathrm{P}_{3}$ & 59 & 81 & 182 & 273 & 120 & 154 & 333 & 491 \\
\hline 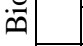 & $\mathrm{P}_{4}$ & 64 & 88 & 195 & 296 & 130 & 166 & 348 & 513 \\
\hline & $\mathrm{P}_{1}$ & 57 & 78 & 169 & 264 & 115 & 147 & 313 & 461 \\
\hline & $\mathrm{P}_{2}$ & 61 & 83 & 183 & 283 & 120 & 154 & 328 & 483 \\
\hline Nี & $\mathrm{P}_{3}$ & 66 & 90 & 198 & 306 & 128 & 165 & 347 & 511 \\
\hline & $\mathrm{P}_{4}$ & 72 & 99 & 212 & 333 & 134 & 171 & 359 & 529 \\
\hline \multicolumn{10}{|c|}{ Bio-fertilizers (Mycorrhizae) } \\
\hline & $\begin{array}{c}\text { Contro } \\
1\end{array}$ & 43 & 59 & 125 & 188 & 80 & 104 & 212 & 319 \\
\hline & $\mathrm{P}_{1}$ & 60 & 82 & 166 & 262 & 120 & 157 & 321 & 483 \\
\hline ฟี & $\mathrm{P}_{2}$ & 66 & 91 & 178 & 289 & 127 & 166 & 339 & 509 \\
\hline & $\mathrm{P}_{3}$ & 69 & 95 & 193 & 302 & 134 & 174 & 362 & 544 \\
\hline 电 & $\mathrm{P}_{4}$ & 75 & 103 & 198 & 328 & 143 & 187 & 373 & 560 \\
\hline & $\mathrm{P}_{1}$ & 65 & 89 & 181 & 284 & 128 & 167 & 329 & 495 \\
\hline & $\mathrm{P}_{2}$ & 71 & 97 & 195 & 310 & 134 & 174 & 358 & 538 \\
\hline $\mathrm{N}$ & $\mathrm{P}_{3}$ & 77 & 106 & 207 & 337 & 142 & 185 & 377 & 566 \\
\hline & $\mathrm{P}_{4}$ & 83 & 114 & 215 & 363 & 151 & 197 & 385 & 578 \\
\hline \multicolumn{10}{|c|}{ Bio-fertilizers (Mycorrhizae+ Azotobacter ) } \\
\hline \multirow{8}{*}{.} & $\begin{array}{c}\text { Contro } \\
1\end{array}$ & 49 & 65 & 132 & 196 & 88 & 113 & 227 & 336 \\
\hline & $\mathrm{P}_{1}$ & 67 & 89 & 175 & 268 & 128 & 166 & 332 & 492 \\
\hline & $\mathrm{P}_{2}$ & 75 & 99 & 184 & 300 & 134 & 173 & 359 & 532 \\
\hline & $\mathrm{P}_{3}$ & 79 & 105 & 197 & 316 & 144 & 187 & 375 & 556 \\
\hline & $\mathrm{P}_{4}$ & 86 & 114 & 208 & 344 & 153 & 197 & 390 & 578 \\
\hline & $\mathrm{P}_{1}$ & 73 & 97 & 188 & 292 & 138 & 178 & 344 & 510 \\
\hline & $\mathrm{P}_{2}$ & 78 & 103 & 195 & 312 & 146 & 188 & 377 & 558 \\
\hline & $\mathrm{P}_{3}$ & 84 & 111 & 206 & 336 & 154 & 199 & 394 & 584 \\
\hline งี & $\mathrm{P}_{4}$ & 93 & 123 & 219 & 372 & 161 & 208 & 404 & 598 \\
\hline \multicolumn{2}{|c|}{$\frac{1}{\operatorname{LSD}_{0.05} \mathrm{Bio}}$} & 3.1 & 3.7 & 2.5 & 6.2 & 4.1 & 5.6 & 7.2 & 11.5 \\
\hline \multicolumn{2}{|c|}{$\mathrm{LSD}_{0.05} \mathrm{Zn}$} & 0.3 & 0.4 & 0.8 & 1.4 & 0.4 & 0.6 & 0.8 & 1.1 \\
\hline \multicolumn{2}{|c|}{$\mathrm{LSD}_{0.05} \mathrm{P}$} & 0.7 & 0.9 & 1.5 & 2.8 & 1.2 & 1.6 & 3.1 & 4.7 \\
\hline \multicolumn{2}{|c|}{$\begin{array}{l}\text { LSD }_{0.05} \text { Biox } \\
\mathrm{Zn}\end{array}$} & 0.4 & 0.6 & 1.1 & 2.0 & 0.6 & 0.8 & 1.1 & 1.6 \\
\hline \multicolumn{2}{|c|}{$\begin{array}{l}\text { LSD }_{0.05} \text { Bio } x \\
P\end{array}$} & 0.9 & 1.2 & 2.2 & 3.9 & 1.7 & 2.2 & 4.4 & 6.6 \\
\hline \multicolumn{2}{|c|}{$\mathrm{LSD}_{0.05} \mathrm{Zn} \times \mathrm{P}$} & 1.1 & 1.5 & 2.7 & 4.8 & 2.1 & 2.7 & 5.4 & 8.1 \\
\hline \multicolumn{2}{|c|}{$\begin{array}{ll}\mathrm{LSD}_{0.05} & 3 \\
\text { factors } & \end{array}$} & 1.2 & 1.6 & 2.8 & 5.1 & 2.2 & 2.9 & 5.7 & 8.5 \\
\hline
\end{tabular}

${ }^{1}$ T: Total, Asc*: $\mu \mathrm{g}$ Ascorbic acid/ml extract, Gal**: $\mu \mathrm{mol}$ of Gallic acid/ml extract

Egypt. J. Soil Sci. 56, No. 1 (2016) 
THE INTEGRATION EFFECT BETWEEN MINERAL ...

TABLE 7 . Effect of mineral and bio-fertilizers applied on total microbial counts, PDB counts and $\mathrm{CO}_{2}$ evolution in rhizosphere and azotobacter densities in phyllosphere of wheat in studied soils.

\begin{tabular}{|c|c|c|c|c|c|c|c|c|c|c|c|c|c|c|c|c|c|c|}
\hline \multirow{4}{*}{\multicolumn{3}{|c|}{ Treatments }} & \multirow{2}{*}{\multicolumn{4}{|c|}{$\begin{array}{c}\begin{array}{c}\text { Total microbial } \\
\text { counts }\end{array} \\
\text { (counts X10 }{ }^{2} \mathrm{CFUg} \\
\left.{ }^{1} \mathrm{D} . \mathrm{S}\right)\end{array}$}} & \multirow{2}{*}{\multicolumn{4}{|c|}{$\begin{array}{c}\text { PDB counts } \\
\text { in rhizosphere } \\
\text { (counts } X_{10}^{2} \mathrm{CFUg}^{-1} \\
\text { D.S })\end{array}$}} & \multirow{2}{*}{\multicolumn{4}{|c|}{$\begin{array}{c}\begin{array}{c}\text { Azotobacter count } \\
\text { in phyllosphere }\end{array} \\
\text { (counts X10 } 0^{2} \mathrm{CFUg}^{-} \\
\left.{ }^{1} \text { F.L }\right)\end{array}$}} & \multirow{2}{*}{\multicolumn{4}{|c|}{$\begin{array}{c}\mathrm{CO}_{2} \text { evolution } \\
\begin{array}{c}\left(\mathrm{mg} \mathrm{100 \textrm {g } ^ { - 1 }} \text { dry soil }\right. \\
\left.24 \mathrm{hr}^{-1}\right)\end{array}\end{array}$}} \\
\hline & & & & & & & & & & & & & & & & & & \\
\hline & & & \multicolumn{2}{|c|}{$\begin{array}{c}\text { Sandy } \\
\text { soil }\end{array}$} & \multicolumn{2}{|c|}{ Clay soil } & \multicolumn{2}{|c|}{$\begin{array}{c}\text { Sandy } \\
\text { soil }\end{array}$} & \multicolumn{2}{|c|}{ Clay soil } & \multicolumn{2}{|c|}{$\begin{array}{c}\text { Sandy } \\
\text { soil }\end{array}$} & \multicolumn{2}{|c|}{ Clay soil } & \multicolumn{2}{|c|}{$\begin{array}{c}\text { Sandy } \\
\text { soil }\end{array}$} & \multicolumn{2}{|c|}{ Clay soil } \\
\hline & & & $\mathbf{S t}_{1}$ & $\mathbf{S t}_{2}$ & $\mathbf{S t}_{1}$ & $\mathbf{S t}_{2}$ & $\mathbf{S t}_{1}$ & $\mathrm{St}_{2}$ & $\mathbf{S t}_{1}$ & $\mathbf{S t}_{2}$ & $\mathbf{S t}_{\mathbf{1}}$ & $\mathbf{S t}_{2}$ & $\mathbf{S t}_{1}$ & $\mathbf{S t}_{2}$ & $\mathbf{S t}_{\mathbf{1}}$ & $\mathbf{S t}_{2}$ & $\mathbf{S t}_{1}$ & $\mathbf{S t}_{2}$ \\
\hline \multicolumn{19}{|c|}{ Mineral fertilizers } \\
\hline \multirow{9}{*}{ 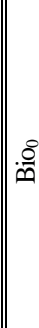 } & & $\begin{array}{c}\text { Contr } \\
\text { ol }\end{array}$ & 24 & 21 & 297 & 240 & 4.4 & 3.8 & 21 & 13 & 2.8 & 2.3 & 3.7 & 3.1 & 26 & 22 & 32 & 27 \\
\hline & & $\mathrm{P}_{1}$ & 37 & 33 & 450 & 364 & 6.6 & 4.7 & 29 & 16 & 3.2 & 2.9 & 3.8 & 3.4 & 32 & 29 & 35 & 32 \\
\hline & & $\overline{P_{2}}$ & 41 & 37 & 529 & 428 & 7.8 & 5.5 & 34 & 19 & 3.5 & 3.2 & 4.3 & 3.9 & 37 & 33 & 41 & 37 \\
\hline & & $\mathrm{P}_{3}$ & 43 & 38 & 599 & 484 & 8.9 & 6.7 & 39 & 23 & 4.0 & 3.6 & 4.9 & 4.4 & 41 & 38 & 47 & 42 \\
\hline & & $\mathrm{P}_{4}$ & 47 & 42 & 663 & 536 & 9.9 & 7.9 & 45 & 27 & 4.4 & 4.0 & 5.4 & 4.9 & 45 & 41 & 52 & 47 \\
\hline & & $\mathrm{P}_{1}$ & 41 & 37 & 495 & 400 & 6.8 & 5.1 & 32 & 17 & 3.4 & 3.1 & 4.2 & 3.8 & 34 & 31 & 39 & 35 \\
\hline & & $\mathrm{P}_{2}$ & 46 & 41 & 604 & 488 & 7.9 & 5.8 & 38 & 20 & 3.9 & 3.5 & 4.7 & 4.3 & 41 & 37 & 47 & 43 \\
\hline & & $\mathrm{P}_{3}$ & 50 & 45 & 673 & 544 & 8.1 & 6.9 & 40 & 24 & 4.3 & 3.9 & 5.3 & 4.9 & 46 & 42 & 2 & 48 \\
\hline & & $\mathrm{P}_{4}$ & 52 & 46 & 717 & 580 & 10.2 & 8.1 & 49 & 28 & 4.9 & 4.5 & 5.8 & 5.3 & 49 & & & 51 \\
\hline \multicolumn{19}{|c|}{ Bio-fertilizers (Mycorrhizae) } \\
\hline \multirow{9}{*}{ a } & & $\begin{array}{c}\text { Contr } \\
\text { ol }\end{array}$ & 32 & 26 & 376 & 304 & 4.8 & 4 & 23 & 14 & 3.1 & 2.6 & 3.9 & 3.2 & 31 & 26 & 41 & 34 \\
\hline & & $\mathrm{P}_{1}$ & 47 & 38 & 539 & 436 & 8.1 & 6.2 & 35 & 21 & 3.8 & 3.5 & 4.2 & 3.8 & 35 & 32 & 42 & 38 \\
\hline & & $\mathrm{P}_{2}$ & 51 & 42 & 613 & 496 & 9.6 & 7.3 & 41 & 25 & 4.4 & 4.0 & 4.9 & 4.5 & 39 & 36 & & 43 \\
\hline & & $\mathrm{P}_{3}$ & 54 & 44 & 698 & 564 & 10.9 & 8.8 & 47 & 30 & 4.9 & 4.5 & 5.5 & 5.0 & 44 & 40 & 54 & 49 \\
\hline & & $\mathrm{P}_{4}$ & 59 & 48 & 777 & 628 & 12.2 & 10.4 & 53 & 36 & 5.4 & 4.9 & 6.1 & 5.6 & 49 & 45 & 60 & 55 \\
\hline & & $\mathrm{P}_{1}$ & 53 & 43 & 599 & 484 & 8.3 & 6.7 & 39 & 23 & 4.1 & 3.7 & 4.6 & 4.2 & 38 & 35 & 77 & 42 \\
\hline & & $\mathrm{P}_{2}$ & 56 & 46 & 678 & 548 & 9.7 & 7.7 & 46 & 26 & 4.8 & 4.4 & 5.6 & 5.1 & 43 & 39 & 53 & 48 \\
\hline & & $\mathrm{P}_{3}$ & 59 & 48 & 762 & 616 & 9.9 & 9.1 & 47 & 31 & 5.5 & 5.0 & 6.2 & 5.7 & 48 & 44 & 59 & 54 \\
\hline & & $\mathrm{P}_{4}$ & 64 & 53 & 831 & 672 & 11.5 & 10.7 & 55 & 37 & 5.8 & 5.3 & 6.6 & 6.0 & 55 & 50 & 65 & 59 \\
\hline \multicolumn{19}{|c|}{ Bio-fertilizers (Mycorrhizae+ Azotobacter) } \\
\hline & & $\begin{array}{c}\text { Contr } \\
\text { ol }\end{array}$ & 38 & 31 & 430 & 348 & 5.4 & 4.7 & 26 & 16 & 41 & 34 & 56 & 47 & 34 & 29 & 47 & 39 \\
\hline & & $\mathrm{P}_{1}$ & 69 & 56 & 648 & 524 & 8.6 & 6.8 & 39 & 23 & 48 & 44 & 60 & 55 & 41 & 37 & 50 & 46 \\
\hline & & $\mathrm{P}_{2}$ & 72 & 60 & 732 & 592 & 10.7 & 8.6 & 48 & 29 & 54 & 49 & 68 & 62 & 46 & 41 & 57 & 52 \\
\hline & & $\mathrm{P}_{3}$ & 80 & 66 & 836 & 676 & 11.4 & \begin{tabular}{|l|}
9.7 \\
\end{tabular} & 54 & 33 & 63 & 57 & 77 & 70 & 53 & 4 & 65 & 59 \\
\hline & & $\mathrm{P}_{4}$ & 87 & 71 & 900 & 728 & 12.4 & \begin{tabular}{|l|}
11.7 \\
\end{tabular} & 58 & 40 & 69 & 63 & 83 & 76 & 58 & 53 & 70 & 64 \\
\hline & & $\overline{P_{1}}$ & 72 & 60 & 683 & 552 & $\begin{array}{l}9.2 \\
\end{array}$ & \begin{tabular}{|l|}
7.9 \\
\end{tabular} & 43 & 27 & 53 & 48 & 63 & 58 & 44 & 40 & 53 & 48 \\
\hline & & $\mathrm{P}_{2}$ & 79 & 65 & 782 & 632 & 10.9 & 9.1 & 52 & 31 & 59 & 54 & 72 & 66 & 50 & 45 & 61 & 55 \\
\hline & & $\mathrm{P}_{3}$ & 85 & 70 & 886 & 716 & 11.2 & \begin{tabular}{|l|}
10.2 \\
\end{tabular} & 55 & 35 & 66 & 60 & 82 & 75 & 56 & 50 & 69 & 63 \\
\hline & & $\mathrm{P}_{4}$ & 92 & 75 & 965 & 781 & 12.9 & \begin{tabular}{|l|}
11.1 \\
\end{tabular} & 59 & 38 & 73 & 67 & 89 & 81 & 62 & 56 & 75 & 68 \\
\hline & & Bio & 5.8 & 4.3 & 36 & 29 & 0.43 & \begin{tabular}{|l|l|}
0.49 \\
\end{tabular} & 2.1 & 1.7 & 11 & 10 & 14 & 12 & 1.9 & 1.7 & 3.0 & 2.7 \\
\hline & & & 0.3 & 0.2 & 3 & 2 & \begin{tabular}{|l|}
0.18 \\
\end{tabular} & \begin{tabular}{|l|}
0.18 \\
\end{tabular} & 0.9 & 0.6 & 0.6 & 0.1 & 0.6 & 0.1 & 0.2 & 0.2 & 0.2 & 0.2 \\
\hline & 05 & & 0.7 & 0.6 & 8 & 7 & \begin{tabular}{|l|l|}
0.10 \\
\end{tabular} & \begin{tabular}{|l|}
0.08 \\
\end{tabular} & 0.4 & 0.3 & $\begin{array}{ll}0.2 \\
\end{array}$ & 0.3 & 0.3 & 0.4 & 0.4 & 0.3 & 0.5 & 0.4 \\
\hline & & & 0.2 & 0.2 & 4 & 3 & 0.25 & 0.25 & 1.3 & 0.9 & 0.8 & 0.2 & 0.8 & 0.2 & 0.2 & 0.2 & 0.3 & 0.2 \\
\hline $\begin{array}{l}\text { LS } \\
P\end{array}$ & & Bio $x$ & 1.0 & 0.8 & 12 & 9 & 0.14 & 0.12 & 0.6 & 0.4 & 0.3 & 0.4 & 0.4 & 0.4 & 0.6 & 0.5 & 0.6 & 0.5 \\
\hline & & $\mathrm{Zn \times P}$ & 1.2 & 1.0 & 14 & 12 & 0.17 & 0.14 & 0.7 & 0.5 & 0.4 & 0.5 & 0.5 & 0.6 & 0.7 & 0.6 & 0.8 & 0.7 \\
\hline & & factors & 1.3 & 1.1 & 15 & 12 & 0.24 & 0.20 & 1.0 & 0.7 & 0.6 & 0.7 & 0.7 & 0.8 & 1.0 & 1.0 & 0.8 & 0.8 \\
\hline
\end{tabular}

$\mathrm{St}_{1}=$ vegetating stage, $\mathrm{St}_{2}=$ harvesting stage, $\mathrm{F} . \mathrm{L}=$ fresh leaf, $\mathrm{D} . \mathrm{S}=$ dry soil, $\mathrm{PDB}=$ Phosphate dissolved bacteria 
Data also showed that the Azotobacter densities from the phyllosphere of wheat plants were 2.8 and $3.7 \times 10^{2} \mathrm{cfu} \mathrm{cm}^{-2}$ leaf in control treatment in sandy and clay soils, respectively. While these values increased to reach 73 and 89 $\times 10^{2} \mathrm{cfu} / \mathrm{cfu} / \mathrm{cm}^{2}$ leaf with treating the plants with $\mathrm{P}_{4}+\mathrm{Zn}_{1}$ along with Mycorrhizae + Azotobacter in sandy and clay soils, respectively. These results agreeable with that obtained by Khin (2011) who reported that mixture of biofertilizer and zinc sulphate gave better results than individual treatments.

\section{$\mathrm{CO}_{2}$ evolution}

The $\mathrm{CO}_{2}$ evolved from rhizosphere $\left(\mathrm{mg} \mathrm{CO} \mathrm{CO}_{2} 100 \mathrm{~g}^{-1}\right.$ dry soil $24 \mathrm{hr}^{-1}$ ) was periodically determined to detect the microbial activity as influenced by mineral $(\mathrm{P}+\mathrm{Zn})$ and bio-fertilizers (Mycorrhizae + Azotobacter) treatments.

Data in Table 7 show that the $\mathrm{CO}_{2}$ evolution increased in rhizosphere reaching maximum levels at 40 days after sowing (vegetation stage) then decreased during harvesting stage in both soils. In both soils, rhizosphere of control treatment (without inoculation with bio-fertilizers) gave the lowest $\mathrm{CO}_{2}$ evolution values. Results also showed that $\mathrm{CO}_{2}$ evolved in rhizosphere of wheat were markedly affected by application of $\mathrm{P}$ and $\mathrm{Zn}$ treatments. Increasing $\mathrm{P}$ and $\mathrm{Zn}$ rates led to increase yield parameters to reach maximum value in the treatment of the $\mathrm{P} 4+\mathrm{Zn} 1 . \mathrm{CO}_{2}$ evolution values in rhizosphere were much higher in clayey soils compared with sandy soils. Bio-fertilizers (Mycorrhizae) and (Mycorrhizae + Azotobacter) treatments increased $\mathrm{CO}_{2}$ evolution in the presence of mineral fertilizers. The most effective treatment was $\mathrm{NP}_{4} \mathrm{~K}+\mathrm{Zn}_{1}$ plus Mycorrhizae + Azotobacter which produced 61.57 and $55.97\left(\mathrm{mg} \mathrm{CO}_{2} 100 \mathrm{~g}^{-1}\right.$ dry soil $24 \mathrm{hr}^{-1}$ ) in vegetation and harvesting stages, respectively, in sandy soil. While produced 75.08 and $68.25\left(\mathrm{mg} \mathrm{CO}_{2} 100 \mathrm{~g}^{-1}\right.$ dry soil $\left.24 \mathrm{hr}^{-1}\right)$, respectively, in clayey soil. These result agreeable with that obtained by Amanullah et al. (2012); Kowsar et al. (2014); and Garshasbi et al. (2014).

\section{Conclusions}

Application of mineral $(\mathrm{P}+\mathrm{Zn})$ and bio-fertilizers (Mycorrhizae+ Azotobacter) increased yield parameters, nutrients contents, nutrients uptake and biochemical components of wheat grown in the New Valley soils with high $\mathrm{Fe}$ content. These parameters were much higher in the clay soils than in the sandy soils. Bio-fertilizers (Mycorrhizae) and (Mycorrhizae+Azotobacter) treatments increased yield parameters of wheat in the presence of mineral fertilizers by increasing the available $\mathrm{N}$ and $\mathrm{P}$ in soils. The most effective treatment appeared to be applying $\mathrm{P}$ at a rate of $60 \mathrm{~kg} \mathrm{fed}^{-1}$ in clay soil and $80 \mathrm{~kg} \mathrm{fed}^{-1}$ in sandy soil with spraying $\mathrm{Zn}$ at a concentration of $250 \mathrm{mg} \mathrm{L}^{-1}$ combined with the application of Mycorrhizae and Azotobacter which achieved 5.45 and $2.21 \mathrm{Mg} \mathrm{fed}^{-1}$ for straw and grains respectively in the sandy soil, while 9.5 and $4.16 \mathrm{Mg} \mathrm{fed}^{-1}$ in the clay soil. This study revealed that the combination of bio- and mineral $\mathrm{P}$ and $\mathrm{Zn}$ fertilizers could decrease the negative effect of high Fe content in soils and increased wheat production.

Egypt. J. Soil Sci. 56, No. 1 (2016) 


\section{Referances}

Abbas, G., Hussain, F. Anwar, Z., Khattak, J.Z.K., Ishaque, M. and Ullah, A. (2012) Effects of iron on the wheat crop (Triticum aestivum L.) by uptake of nitrogen, phosphorus and potassium. Asian Journal of Agricultural Sciences 4(3) 229-235, 2012 .

Abbas, G., Khan, M.Q. Khan, M.J., Tahir, M., Ishaque, M. and Hussain, F. (2011) Nutrient uptake, growth and yield of wheat (Triticum aestivum 1.) as affected by manganese application. Pak. J. Bot., 43(1), 607-616, 2011.

Abbas,G., Khattak, J.Z.K. Abbas, G., IShaque, M., Aslam, M., Abbas, Z., Amer, M. and Khokhar, M.B. (2013) Profit maximizing level of potassium fertilizer in wheat production under arid environment. Pak. J. Bot., 45(3), 961-965.

Amanullah, A.A.K., Saifullah, K. Munir, A. and Khan, J. (2012) Biofertilizer- a possible subsitute of fertilizers in production of wheat variety zardana in balochistan. Pakistan J. Agric. Res. 25 (1).

Barker, J. S. and Tagu, D. (2002) The role of auxins and cytokinins in mycorrhizal symbioses. J. Plant Growth Regul. 19,144-154.

Castillo C.G., Puccio, F. Morales, D., Borie, F. and Sieverding, E. (2012) Early arbuscular mycorrhiza colonization of wheat, barley and oats in Andosols of southern Chile. J. of Soil Sci. and Plant Nutr., 12 (3), 511-524.

Cottenie, A., Verlso, M. Kilkens, L., Velghe, G. and Camerlynck, R. (1982) Chemical Analysis of Plants and Soils. Lab. Agroch. State Univ. Gent, Belgium.

Ehsan, S., Shahid, J. Ifra, S., Fareeha, H. and Majeed, T. (2014) Effect of humic acid foliar spraying and nitrogen fertilizers management on wheat yield. International Journal of Agronomy and Agricultural Research, 4(4), 28-33.

Ewa S.G., and Jolanta, K. (2005) Effect of excessive zinc content in soil on the phosphorus content in wheat plants. Electronic Journal of Polish Agricultural Universities, Environmental Development, Topic, Issue 4, Volume 8.

Fadl-Allah, E.M., El-komy, H. M. and Sholkamy, E.N. (2010) The effect of cyanobacteria, nostoc muscorum as biofertilizer on growth and n-yield of wheat (Triticum aestivum 1.) under different doses of $\mathrm{n}$-fertilizer. Journal of the Association of Arab Universities for Basic \& Appl; 8, p61.

Garshasbi, L., Paknejad, F., Kashani, A., Nateghi, M. and Fatemi, Z. (2014) Wheat performance as affected by pre-plant and foliar application of phosphorus under inoculation with arbuscular mycorrhizal (AM) and non-inoculation. International Journal of Biosciences, 4(5),154-161.

Gerdemann, J. W. and Nicolson, T. H. (1963) Spores of mycorrhizal Endogone species extracted from soil by wet sieving and decanting. Trans. Brit. Mycol. Soc. 46, $235-$ 244.

Gomez, K.A. and Gomez, A.A. (1984) Statistical Procedures for Agricultural Research. 2nd ed., Wiley, New York.

Handreck, K.A. (2006) Interactions between iron and phosphorus in the nutrition of Banksia ericifolia L.f var ericifolia (Proteaceae) in soil-less Potting media. Australian Journal of Botany 39(4) 373-384. 
Hasanpour J., Panahi, M.S. P. M. M. and Arabsalmani, K. (2012) Effect of inoculation with VA mycorrhiza and Azotobacter on grain yield, LAI and protein of wheat on drought stress condition. Inter. J. of Agri Sci. 2(6), 466-476.

Hasina, G., Ahmad, S., Beena, S., Fida, M. and Ahmad, I. (2011) Effect of foliar application of nitrogen, potassium and zinc on wheat growth. ARPN Journal of Agricultural and Biological Science, 6(4), ISSN 1990-6145.

Hill, G. Sawers,(2000) Azotobacter. In Encyclopedia of Microbiology $3^{\text {rd }}$ ed, Academic Press, New York, 2000, pp.359-371

Kefyalew G., Martin, K. L., Freeman, K. W., Mosali, J., Teal, R. K., Raun, W. R., Moges, S. M. and. Arnall, D. B. (2006) Determination of Optimum Rate and Growth Stage for Foliar Applied Phosphorus in Corn. Department of Plant and Soil Sciences, Oklahoma State University, Stillwater, Ok, 74078, Contribution from the Oklahoma Agricultural Experiment Station.

Khin S. Aye (2011) Investigation on the effectiveness of zinc sulphate and biofertilizer on mustard plant. Inter. Scholarly and Scientific Research \& Innovation 5(3), 233-235.

Klute, A.A. (1986) Methods of Soil Analysis. Part $12^{\text {nd }}$ ed. American Society of Agronomy .Inc. Publishes, Madison, Wisconsin, USA.

Kowsar, J., Rather, A.M. Boswal, M.V. and Ganie, A.H. (2014) Effect of biofertilizer and organic fertilizer on morpho-physiological parameters associated with grain yield with emphasis for further improvement in wheat yield production (Bread INheat=Triticum aestivum L.). International J. of Agric. and Crop Sci.,7(4),178-184.

Lachman, J., Miholová, D. Pivec, V., Jírů, K. and Janovská, D. (2011) Content of phenolic antioxidants and selenium in grains of einkorn (Triticum monococcum), emmer (Triticum dicoccum) and spring wheat (Triticum aestivum) varieties. Plant Soil Environ., 57(5), 235-243.

Li, B.Y., Zhou, D.M., Cang, L., Zhang, H.L., Fan, X.H. and Qin, S.W. (2007) Soil micronutrient availability to crops as affected by long-term inorganic and organic fertilizer applications. Soil and Tillage Research, 96 (1-2),166-173.

Li, Y., Sun, H., Li, H., Yang, L., Ye, B. and Wuyi, W. (2013) Dynamic changes of rhizosphere properties and antioxidant enzyme responses of wheat plants (Triticum aestivum L.) grown in mercury-contaminated soils. Chemosphere, http://dx. doi.org 10.1016/j.chemosphere, 50-63.

Mikhail, Karima, K.(2002) Environmental factors affecting microbial activities in the phyllosphere of some medicinal desert plants. Ph.D. Thesis, Institute of Environmental Studies and Research, Ain Shams Univ., Cairo, Egypt.

Mir Reza Miri, Moghadam, H. R. T., Ghooshchi, F., Zahedi, H. (2013) Effect of Azotobacter and Arbuscular Mycorrhizal colonization enhance wheat growth and physiological traits under well-watered and drought conditions. Advances in Environ. Biol. 7(14), pp. 4630-4636.

Moghadam, M.J., Sharifabad1, H.H. , Noormohamadi1, G., Motahar, S.Y.S. and Siadat, S.A. (2012) The effect of zinc, boron and copper foliar application, on yield and yield components in wheat (Triticum aestivum). Annals of Biological Research, $\mathbf{3}$ (8),3875-3884

Moola,R., Davari, M. R. and Sharma, S.N. (2014) Direct, residual and cumulative effects of organic manures and biofertilizers on yields, NPK uptake, grain quality and

Egypt. J. Soil Sci. 56, No. 1 (2016) 
economics of wheat (Triticum aestivum L.) under organic farming of rice-wheat cropping system. Journal of Organic Systems, 9(1)16-30.

Nadim, M. A., Awan, I.U., Baloch, M.S., Khan, E.A., Naveed, K., Khan, M.A., Zubair, M. and Hussain, N. (2011) Effect of micronutrients on growth and yield of wheat. Pak. J. Agri. Sci., Vol. 48(3), 191-196,2011.

Nautiyal, C.S. (1999) An efficient microbiological growth medium for screening phosphate solubilizing microorganisms. FEMS Microbiology Letters, 170, 265 - 270.

Neetu, N., Aggarwal, A., Tanwar, A. and Alpa, A. (2012) Influence of arbuscular mycorrhizal fungi and Pseudomonas fluorescens at different superphosphate levels on linseed (Linum usitatissimum L.) growth response. Chilean Journal of Agricultural Research 72 (2), 237-243.

Niemi, S.N.A.L., Dohuki, M.S.S. and Barwary, S. S. (2014) Combined effect of iron and wheat genotype on seedling tissue concentration of $\mathrm{Fe}, \mathrm{Zn}, \mathrm{Cu}$ and $\mathrm{Mn}$. Journal of Agri-Food and Applied Sciences. Available online at jaas.blue-ap.org (02014 JAAS Journal. 2(3), 60-66.

Page, A.L., Miller, R.H. and Keeney, D.R. (1984) Methods of Soil Analysis. Part 2: Chemical and Microbiological Properties. Second edition. Agronomy J. 9: 2, Am. Soc. Agron. Inc., Soil Sci. Soc. Am. Inc. Pub. Madison, Wisconsin, USA.

Pramer, D. and Schmidt, E.L. (1964) Experimental Soil Microbiology, Burgess Pub., Co., Minneota, USA.

Rimmer; Smith (2009) Antioxidants in soil organic matter and in associated plant materials. European Journal of Soil Science, 60, No. 2, April 2009, pp. 170-175(6).

Ríos J.J., Blasco, B., Cervilla, L.M., Rosales, M.A., Sanchez-Rodriguez, E., Romero, L. and Ruiz, J.M. (2009) Production and detoxification of $\mathrm{H} 2 \mathrm{O} 2$ in lettuce plants exposed to selenium. Annals of Applied Biology, 154,107-116.

Shah, A. S., Shah, S. M., Mohammad, W., Shafi, M., Nawaz, H., Shehzadi, S. and Amir, M. (2010) Effect of integrated use of organic and inorganic nitrogen sources on wheat yield. Sarhad J. Agric. 26(4), 559-563.

Singhal, S.K., Sharma, V.K. and Singh, R.D. (2012) Effect of inorganic and biofertilizers (vam and psb) on yield of maize and wheat cropping sequence and soil fertility. Indian Journal of Agricultural Research; 46(2) p167.

Tahir, M., Ayub, M. Javeed, H.M.R., Naeem, M., Rehman, H.U., Waseem, M. and A.Muqarrab (2011) Effect of Different Organic Matter on Growth and Yield of Wheat (Triticum aestivum L.). Pak. J. Life Soc. Sci., 9(1), 63-66.

Tayebeh, A., Alemzadeh, A. and Kazemeini, S.A. (2010) Effect of organic and inorganic fertilizers on grain yield and protein banding pattern of wheat. Australian Journal of Crop Science (AJCS) 4(6), 384-389.

Vaher, M., Matso, K., Levandi, T., Helmja, K. and Kaljurand, M. (2010) Phenolic compounds and the antioxidant activity of the bran, flour and whole grain of different wheat varieties. Procedia Chemistry, 2(1),76-82.

Wandruszka, Ray von (2006) Phosphorus retention in calcareous soils and the effect of organic matter on its mobility. Department of Chemistry, University of Idaho, Moscow, ID 83844-2343, USA, Geochemical Transactions, 7:6. 
Yassen, A.1., Abou El-Nour, E.A.A. and Shedeed, S. (2010) Response of Wheat to Foliar Spray with Urea and Micronutrients. Journal of American Science 2010; 6(9),14-22.

Yoshie, M., Marjorie Reyes-Díaz and Maria de la Luz Mora Gil (2008) Effect of selenite on the total polyphenol content and antioxidative activity of aqueous and ethanolic extracts in sprouts of four agronomic species. J. Soil Sc. Plant Nutr. 8 (1), $55-67$.

(Received 15/4/2015; accepted 17/5/2015)

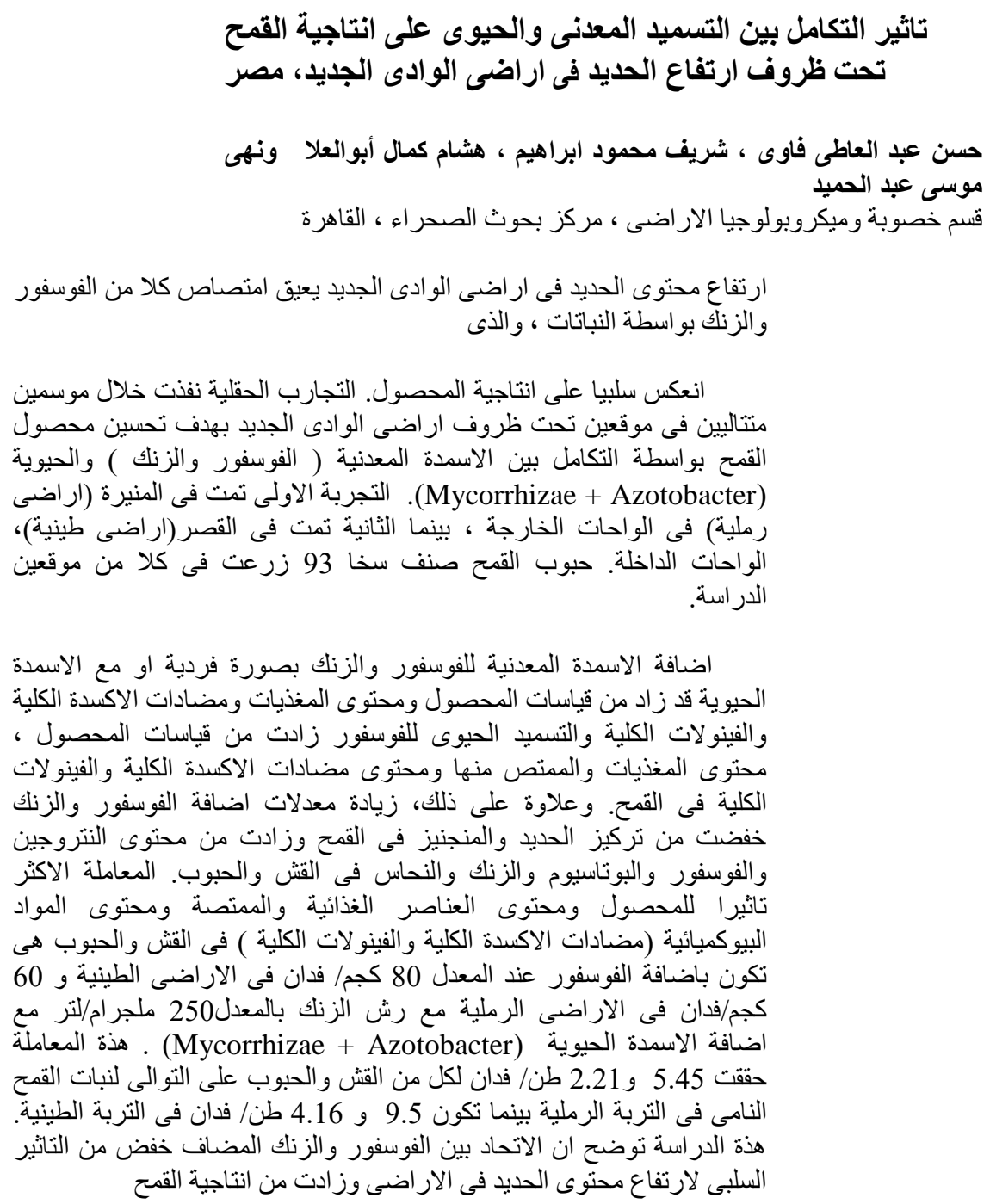

